\title{
Correction to: Building Behavioral Health Homes: Clinician and Staff Perspectives on Creating Integrated Care Teams
}

\author{
Tracy Anastas, BA \\ Elizabeth Needham Waddell, PhD \\ Sonya Howk, MPA:HA \\ Mark Remiker, MA, CCRP \\ Gretchen Horton-Dunbar, BA \\ L. J. Fagnan, MD
}

\section{Correction to: The Journal of Behavioral Health Services \& Research https://doi.org/10.1007/s11414-018-9622-y}

The Publisher regrets that due to a malfunction in production, there are duplications and errors in the author affiliations of the published article. The correct author affiliations are listed below.

Address correspondence to Elizabeth Needham Waddell, PhD, OHSU-PSU School of Public Health, Oregon Health \& Science University, 3181 SW Sam Jackson Park Rd, MC GH230, Portland, OR 97239, USA.

Tracy Anastas, BA, Oregon Rural Practice-based Research Network (ORPRN), Oregon Health \& Science University, Portland, OR, USA.

Sonya Howk, MPA:HA, Oregon Rural Practice-based Research Network (ORPRN), Oregon Health \& Science University, Portland, OR, USA.

Mark Remiker, MA, CCRP, Oregon Rural Practice-based Research Network (ORPRN), Oregon Health \& Science University, Portland, OR, USA.

L. J. Fagnan, MD, Oregon Rural Practice-based Research Network (ORPRN), Oregon Health \& Science University, Portland, OR, USA.

Gretchen Horton-Dunbar, BA, OHSU-PSU School of Public Health, Oregon Health \& Science University, Portland, OR, USA.

The online version of the original article can be found at https://doi.org/10.1007/s11414-018-9622-y

Journal of Behavioral Health Services \& Research, 2018. 544. (c) 2018 National Council for Behavioral Health. DOI 10.1007/s11414-018-9628-5 